\title{
Place du Centre de Loisirs Sans Hébergement (CLSH) dans une éducation relative au(x) territoire(s)
}

Henri Labbé

\section{(Q) OpenEdition \\ Journals}

Édition électronique

URL : http://journals.openedition.org/ere/4265

DOI : $10.4000 /$ ere.4265

ISSN : 2561-2271

Éditeur

Centr'ERE

Référence électronique

Henri Labbé, « Place du Centre de Loisirs Sans Hébergement (CLSH) dans une éducation relative au(x) territoire(s) », Éducation relative à l'environnement [En ligne], Volume 5 | 2005, mis en ligne le 20 novembre 2005, consulté le 21 février 2020. URL : http://journals.openedition.org/ere/4265 ; DOI 10.4000/ere.4265 


\title{
Place du Centre de Loisirs Sans Hébergement (CLSH) dans une éducation relative au(x) territoire(s)
}

\author{
Henri Labbé
}

Parce que l'un des objectifs essentiels de l'éducation est de favoriser l'insertion sociale des enfants et des jeunes et leur accès à l'autonomie,

l'éducation et les actions éducatives doivent contribuer à créer l'appartenance et à construire nos représentations vis-à-vis de nos territoires de vie. (Jacquemain, 1998)

1 En ce sens, le territoire est un enjeu pour l'action éducative et une entrée pour l'éducation relative à l'environnement ou inversement. Peut-on alors parler " d'éducation relative au territoire»?

2 En complément à la famille et à l'école, le CLSH (Centre de Loisirs sans Hébergement) est bien souvent un des premiers maillons de la politique éducative «enfance/ jeunesse » d'un territoire communal ou intercommunal. Par ailleurs, en France, une nouvelle réglementation des centres de vacances et de loisirs incluant le $\mathrm{CLSH}^{1}$ institue, à partir de mai 2003, la présence dans les déclarations d'accueil, d'un projet éducatif et de son document d'application, le projet pédagogique. Nous considérerons le projet d'action comme l'aboutissement des deux précédents.

3 Nous avons voulu savoir dans un premier temps quelle place occupait l'éducation au territoire dans les CLSH, au sein de ces projets. Peut-on lire dans ces documents puis dans les actions, les spécificités et l'identité du territoire environnant le centre ? N'y a$\mathrm{t}$-il pas des projets «hors sol »? Les résultats d'entretiens visant à répondre à ces questions seront présentés ici. Dans un second temps, à partir d'un accompagnement de quelques centres, nous avons voulu expérimenter et observer, directement sur le terrain, quelques pistes permettant de mieux prendre en compte le territoire du centre dans les trois types de projets énoncés ci-dessus. Ces trois entrées seront illustrées de 
façon non exhaustive par quelques exemples. Dans un troisième temps, nous interrogerons de façon plus globale cette idée d'une éducation relative au(x) territoire(s) et la place que le CLSH peut y occuper.

4 Ce qui est présenté ici n'est donc pas une étude scientifique. Ce sont surtout des pistes pour s'interroger et réfléchir à la notion d'éducation relative aux territoires à propos d'une structure «non spécialisée » en éducation à l'environnement, le CLSH.

\section{Une première série d'entretiens, quelques hypothèses de travail}

5 À partir de cette idée, nous nous sommes attachés durant deux étés (2001 et 2002) à interroger dans le département d'Ille et Vilaine, plus de 20 directeurs de CLSH au sujet de leur centre et de sa relation au territoire ${ }^{2}$. Chaque rencontre sur site a permis d'échanger sur les acteurs et leur relation au milieu et sur l'ancrage local des projets. Notons d'entrée de jeu que ces entretiens se sont déroulés en milieu urbain et en milieu rural et que les résultats ne montrent aucune différence entre ces deux types de milieux.

Signalons que la première idée de ces échanges était d'évoquer l'éducation à l'environnement dans les Centres de Vacances et de Loisirs (CVL) en préparation d'un colloque national sur ce thème avec la Jeunesse au Plein Air (JPA, 2001) et le ministère de la Jeunesse et de Sports. Lors des premiers contacts téléphoniques pour les prises de rendez-vous, il est apparu que le terme « éducation relative à l'environnement » $(\mathrm{ErE})$ évoquait chez les répondants un domaine spécialisé avec des intervenants spécifiques. Ainsi, il a été convenu de parler plutôt d'éducation relative au territoire et cette notion a posé moins de problèmes de compréhension ${ }^{3}$. Certes, des actions d'ErE existent, mais il faut les rechercher dans l'organisation de la vie au quotidien, un rallye " patate ", une sortie à vélo ou un minicamp, par exemple.

7 Cette série d'entretiens a montré que le centre de loisirs est davantage conçu comme un outil d'éducation dans le territoire qu'un outil d'éducation au territoire. Le territoire, comme le milieu ou l'environnement, est perçu comme un support d'action, mais les finalités éducatives n'apparaissent pas dans les écrits. Nous sommes en présence de l'environnement objet d'étude. C'est en tout cas la représentation qu'en ont les directeurs de CLSH. Les projets éducatifs et les projets pédagogiques, exceptés ceux des associations spécialisées (centre de classe verte ou association d'environnement) n'utilisent pas les termes d'écocitoyenneté, respect de l'environnement, gestes au quotidien, développement durable, etc. On trouvera surtout les termes généraux suivants: autonomie de l'enfant, liberté du choix des activités, accès à des pratiques culturelles diversifiées, insertion sociale, intégration, etc. Ces termes apparaissent souvent seuls et sans lien avec l'espace géographique considéré. À la lecture du projet éducatif, voire pédagogique, on ne sait pas s'il est écrit à Saint Malo, Rennes, Crevin, Botmeur, Lille ou Grenoble! Des responsables le reconnaissent. Le risque alors est grand de développer des projets non reliés à l'identité de la commune ou du territoire de vie. J'ai osé utiliser le mot de "projet hors sol» et pourquoi pas "culture hydroponique »! Cependant, il est apparu que lorsqu'un CLSH est intégré à une dynamique locale, cet ancrage est plus visible et facilité. On perçoit alors les prémisses d'une politique éducative locale : les CEL (Contrat Éducatif local), les contrats 
temps libre ou enfance de la CAF (Caisse d'Allocation Familiale), les volets éducatifs des chartes d'environnement ou encore les politiques de mise en valeur du patrimoine (tourisme et loisir compris) sont des vecteurs de cet ancrage local. Se pose alors la question de la cohérence entre ces secteurs : le partenariat existe-t-il ?

8 Notre enquête a ainsi montré que la relation du CLSH à son milieu est plutôt d'ordre social : les enfants, les acteurs et intervenants, les locaux et leurs abords. À l'inverse, le cadre de vie, l'environnement rural, urbain ou le quartier, la culture ou le patrimoine local, l'espace ou la géographie des lieux, ne sont abordés que comme moyens ou lieux de pratiques. Rarement ces éléments sont cités et exploités comme provenant d'un diagnostic ou d'un contexte de base pouvant enrichir et donner du sens aux projets éducatifs et pédagogiques ou encore permettre une articulation entre l'organisateur, l'association, le directeur, etc. Rarement encore, cette relation au territoire est citée comme cadre d'action transversale, porteuse de démarches de projets : éducation à l'environnement ou éducation scientifique, médiation culturelle et mise en valeur des patrimoines, éducation physique, cheminements et approches ludiques. À une époque où l'on parle d'enrichissement des projets éducatifs, voilà des pistes à explorer de nouveau et à exploiter.

9 À la suite de ce premier regard et afin d'approfondir cette idée d'éducation relative aux territoires, un appel à contribution a été lancé dans le cadre de mes missions à la Direction Régionale et Départementale de la Jeunesse et des Sports (DRDJS) de Bretagne. Une fiche technique a été réalisée définissant les objectifs et l'organisation de cette opération. Cette fiche n'a pas été envoyée à tous les CLSH d'Ile et Vilaine, mais elle a été progressivement proposée aux structures déjà en relation avec la DRDJS, dans le cadre des projets « 1000 défis pour ma planète » ou " Écologestes » (voir plus loin), ou lors de visites de CLSH ou CEL, par exemple.

Il s'est agi, avec les CLSH intéressés, de repérer des problématiques et des richesses patrimoniales spécifiques à exploiter. Il a aussi été question d'identifier des acteurs, des partenaires et des moyens à solliciter. Enfin, des orientations et des projets d'action en terme d'ErE et de découverte des patrimoines ont été envisagés: écocitoyenneté, appartenance, appropriation de l'espace, actions dans la vie quotidienne ou sur le cadre de vie, etc.

11 Nous avons offert un accompagnement de quelques demi-journées aux CLSH fonctionnant à l'année. Il s'agissait, à partir d'une prise en compte du contexte spécifique et de l'existant, de réfléchir et d'échanger, mais aussi de travailler sur des documents, d'effectuer des visites ou des actions de terrain ou encore d'analyser des projets existants : rencontres, sentier pédagogique, aménagement d'espace, exposition, etc. Les premiers retours ont été classés en trois types ou trois entrées : projet éducatif, projet pédagogique ou projet d'action.

12 Les exemples qui suivent concernent les secteurs enfance ( $7 / 12$ ans). Il faut remarquer que l'ensemble des territoires concernés sont ruraux ou concernent des communes proches de Rennes.

\section{Entrée par le diagnostic ou le projet éducatif}

13 À Langon, dans le pays de Redon, je participe à un comité de pilotage coordonnant le contrat éducatif local. En amont de ce contrat, un projet éducatif issu d'un diagnostic 
participatif a été réalisé sur quatre communes par la Fédération des foyers ruraux des pays de Vilaine pour le SIVU4 "Quatre à quatre». Parmi les trois orientations de ce projet, la première consiste à " permettre une meilleure appropriation du territoire ».

Cette approche revêt de multiples aspects: historiques, humains, naturels, patrimoniaux, sociaux, etc. Elle se situe cependant plus sur le champ de l'affectif que sur celui du savoir : il s'agit d'aimer le territoire, de s'y sentir bien, plus que de l'apprendre. Elle nécessite pour les animateurs de faire un travail d'écoute et de mise en place de collaborations avec les habitants, les associations, des personnes ressources, etc. Elle doit contribuer au renforcement des liens sociaux et permettre ainsi aux centres de loisirs de participer à la dynamique locale et d'être reconnus comme tels.

Les deux autres orientations consistent à " encourager l'ouverture et l'éveil culturel » et « développer l'esprit citoyen pour la construction de projets collectifs ». Quand on considère par exemple la dynamique de l'école de musique traditionnelle du pays de Redon et son ouverture sur l'interculturel ou encore les nombreux événements où les enfants et les jeunes sont acteurs, on se dit que la relation au territoire est annoncée et que le projet est exemplaire! Le projet de création de jeux traditionnels et surdimensionnés initié par l'association des parents d'élèves est interpellé par cette identité territoriale ; les quilles vont se transformer en menhirs, car la commune recèle d'immenses trésors archéologiques; les accessoires du mini-golf seront des maisons traditionnelles ou la chapelle Ste Agathe, par exemple. Le CLSH en complément avec l'école est à l'œuvre. On s'achemine vers une fête de l'école, mais aussi une fête du jeu avec plusieurs acteurs locaux. On voit ici l'effet immédiat des orientations éducatives $\mathrm{du}$ projet éducatif, mais la construction reste intellectuelle et doit être rappelée en permanence. Que dit le projet éducatif, est-on en cohérence avec celui-ci ? La passion de construire des activités avec les associations doit être interrogée à chaque fois et puis l'école a son projet d'école! L'action en commission est ici primordiale: on construit à plusieurs, c'est la clé de la réussite pour que les actions soient en cohérence avec les orientations éducatives énoncées au départ. C'est aussi l'esprit du CEL.

\section{Entrée par le projet pédagogique et l'action sur une année}

Voici l'entrée qui a été la plus sollicitée et l'appel à contribution y a trouvé naturellement sa place pour l'accompagnement d'opérations déjà existantes. On citera ici le cas de quatre CLSH qui sont associés à la création d'un jardin, c'est-à-dire à l'appropriation d'un espace proche des enfants : leur cadre de vie, leur environnement quotidien ou des pratiques de leurs parents ou grands-parents. Un autre point commun est leur labellisation " 1000 défis pour ma planète $»^{5}$; ces projets sont des actions pour et sur l'environnement avec des effets sur nos comportements écocitoyens. Cette labellisation provoque une certaine fierté des enfants et une reconnaissance autour d'eux. Enfin ces quatre projets sont inscrits dans "l'aventure écologestes » : en un an, créer un jeu sur le thème des plantes et de la végétation. Ainsi, le jeu du UNO sur les plantes se réfère au paysage habituel des enfants tout comme le jeu coopératif abordant le jardin dans un cadre de vie urbain. Que dire du jeu de l'oie sur l'alimentation et l'énergie abordant la diététique, et des activités à la cantine et durant le temps périscolaire. Les réactions sont unanimes quant à la richesse d'un projet mené sur une année entière, sur son territoire et au quotidien. L'entrée par le jeu semble modifier les 
rapports au savoir des animateurs et des enfants ; une journée de formation pose les bases de la démarche. Les jeux réalisés peuvent être prêtés entre CLSH et alimenter un début de réseau. De ces quatre exemples, on soulignera l'ancrage local par une action pérenne sur l'environnement au quotidien des enfants.

Ici, la spécificité du centre de loisirs est certaine notamment au plan de l'action directe sur son espace proche. Il a fallu des dispositifs ou des appels à projets pour initier tout cela. On doit cependant réfléchir au mode de développement plus ou moins contractuel de tous ces outils. N'y a-t-il pas un risque de formatage? Quelle durée? Quel partenariat est provoqué? Que gagne-t-on? Pour les quatre exemples cités, mentionnons que l'opération "écologestes ${ }^{6}{ }^{\prime}$ 'apporte aucun financement particulier sinon un accompagnement en personnel discret et à la demande. On soulignera enfin dans ces exemples que l'entrée par le projet pédagogique implique la coordination de plusieurs actions qui s'enrichissent et s'imbriquent les unes dans les autres, au lieu d'un empilement lourd et non cohérent ; ici, le jardin et la création d'un jeu ou plus encore, l'intégration du jeu sur l'alimentation dans le projet périscolaire qui comprend lui aussi des actions liées à la vie de la cantine (semaine du goût, délégué enfant du midi, art déco et la cantine idéale, etc.) ou encore la rencontre avec une maison de retraite proche ou la création de liens avec un salon « bio » connu.

\section{Entrée par le projet d'action}

Quelques CLSH ont choisi cette piste: un contexte précis va déclencher l'action, la rencontre de personnes ou d'acteurs et la démarche de projet. Un exemple mérite d'être souligné notamment par ses caractéristiques sur le plan du partenariat local. J'ai proposé d'associer deux CLSH pour enrichir une démarche d'aménagement d'une vallée humide par la communauté de communes en zone de loisirs et d'interprétation du paysage. Après une phase de sensibilisation des enfants à cette zone humide (sorties nature, ateliers peinture, rallyes dans les bourgs, diaporama sur les sentiers pédagogiques, visites de sites d'interprétation, etc.), une deuxième phase de créativité est lancée : minicamp environnement avec création d'une pièce de théâtre, invention d'une légende et sa mise en scène. La troisième phase a permis la réalisation par les enfants et les deux équipes d'animateurs d'un rallye découverte dans la zone humide pour les enfants et les familles du canton. Un dernier temps permet à présent d'intégrer les réalisations des enfants dans les dispositifs pédagogiques: bornes interactives, légende du site, dessins d'enfants, livrets, etc. Parallèlement, une phase «rebond» se met en place pour y associer à présent les différentes écoles de la communauté de communes. J'ai accompagné ce projet qui m'a paru pluridisciplinaire et multipartenarial. Cette action a rendu les enfants acteurs et fiers de leur territoire avec une envie de le communiquer. On doit souligner un riche travail d'équipe entre des services techniques municipaux, un animateur spécialisé et les équipes d'animatrices des CLSH. Que dire encore? Laissons parler un élu: Je ne pensais pas que des CLSH puissent être capables de cela! Puis, deux enseignantes non associées au projet: Avec $\mathrm{X}$, toute la classe connaît à présent les différences entre un crapaud et une grenouille ; avec $Y$, le trajet en car n'a pas suffi pour qu'il nous raconte la légende de Monthault. Et pour finir, les enfants : Avec la Malle Ricochet, ce qui est bien, c'est que l'on construit et on invente son propre paysage ; je suis content, car le projet de la zone humide se passe sur ma commune ; je me suis fait deux copains de l'autre centre de loisirs. 
18 Dans l'entrée par le projet d'action, la démarche de projet et le partenariat sembleraient être deux caractéristiques majeures : on construit collectivement et ainsi, on ne sait pas forcément ou on ne programme pas d'avance tout ce qui va se passer ; un événement est prévu, le rallye dans notre exemple, mais tout le reste est une surprise : ainsi, la légende mise en scène par un plasticien ou l'aide apportée par une troupe de théâtre pendant le minicamp. Projet exemplaire autant qu'imprévisible! On a tous connu ce type de projet: une personne au départ qui en rencontre une autre, la mayonnaise prend (ou pas !) et la synergie s'enclenche. On n'aurait jamais pensé que... Mais que se passera-t-il quand les personnes seront parties? Expérience fortuite ou démarche de projet ? En tout cas, ils l'ont fait, c'est possible. Tirons-en les conclusions et mettons en relation une politique environnementale, une politique enfance et jeunesse, le tourisme et le patrimoine, l'école, connectons des boîtes si souvent séparées.

\section{Éducation relative au(x) territoire(s) et $\mathrm{CLSH}$}

De nombreuses pistes de réflexion et de questionnement ont été présentées jusqu'ici.

Le mot territoire est très à la mode actuellement. Il est surtout au cœur de nombreux enjeux actuels: décentralisation et nouvelles compétences territoriales, pays et schémas d'aménagement, politiques éducatives territoriales et cohérence des acteurs, mais aussi, mondialisation culturelle et économique, conflits et territoires occupés, histoire ou avenir. Alors méfions-nous aussi d'un repli identitaire et de cette notion de frontière ou d'exclusion. Et ils sont bien nombreux «nos" territoires de vie: migrations quotidiennes et pendulaires, voyages et grandes vacances, rêves et jardins secrets, tout au long de la vie. Et puis il s'agit d'une véritable appropriation de son territoire à la fois affective, cognitive, éthique et participative. On pourrait aussi parler d'espace conçu, perçu et vécu comme le développe Lefèvre (1974).

Peut-on parler alors d'éducation aux territoires? De toute façon, il nous faut « consolider le lien qu'inévitablement chacun noue, dès sa naissance, avec un territoire dont les frontières évoluent au gré du développement de la personne, du couffin du nouveau-né au village planétaire de l'écocitoyen » (Larue, 1998). Il ne s'agit pas ici de substituer le mot territoire au mot environnement, mais plutôt de souligner la dimension «écosociosystémique » de l'ErE en y incluant les dimensions d'un développement que certains appellent «durable», mais aussi le domaine de la médiation culturelle et la mise en valeur des patrimoines, la préservation et le développement des arts et des traditions populaires, notre environnement quotidien, etc.

Il y a plus de vingt ans que la Fédération nationale des foyers ruraux a développé ses projets en intégrant à la fois les problématiques des espaces ruraux et les enjeux éducatifs qui y sont liés : entre autres, l'exode du milieu rural, le problème d'accès aux loisirs et le maintien de services et accueils éducatifs. Les actions décrites favorisent bien cet ancrage local, cette relation au territoire, cette participation au développement de pays. Les domaines de l'environnement et des patrimoines y inspirent des activités, mais aussi, ils donnent du sens aux questions de territoire et d'éducation. Une éducation POUR et PAR les territoires ruraux. Et la ville alors ? Voilà maintenant plus de dix ans que le réseau national «Citéphile » est né ; le concept d'EEU (éducation à l'environnement urbain) traduit selon moi la territorialisation d'une 
action éducative; il s'agit d'éduquer un citadin citoyen pour mieux vivre la ville. On trouvera de nombreux exemples de projets dans les actes des rencontres nationales (entre autres, Citéphile, 1998).

\section{Alors, quelle place pour le CLSH dans cette éducation relative aux territoires?}

23 Tout d'abord, une distinction doit être faite entre un CLSH acteur de son territoire et un CLSH, outil d'éducation aux territoires. Le CLSH acteur de son territoire, c'est possible: des directeurs de centre et des associations le revendiquent; les premiers entretiens de notre enquête l'ont montré. Plusieurs éléments sont mis en avant: partenariats locaux, réseaux de fournisseurs, emplois, etc. C'est sûr, le CLSH, au plan organisationnel et au regard du développement local, possède un ancrage territorial. Les différents contrats et politiques éducatives territoriales participent de cela. Certains parlent même d'acteur du développement durable. Ceci est une idée récente, des réseaux y agissent et les dernières rencontres nationales sur les CVL (Centres de Vacances et de Loisirs), organisées par la JPA (Jeunesse au Plein Air) et le MJSVA (Ministère de la Jeunesse et des Sports et de la Vie Associative) ont approfondi le thème de l'alimentation et de la consommation sous cet angle du territoire et en tenant compte des spécificités de ces temps de vie particuliers aux CVL (JPA, 2001).

Concernant cette deuxième idée du CLSH acteur d'éducation aux territoires, nous avons avancé des éléments et décrit des exemples à partir de trois portes d'entrée. Des atouts et des contraintes apparaissent. Les projets d'action existent; ils sont surtout développés par les associations dites d'environnement ou spécialisées. Ils sont ponctuels dans le monde des associations généralistes. Les entrées par les projets éducatifs et pédagogiques sont plus riches, plus permanentes, plus cohérentes, mais restent rares. En lisant les projets éducatifs et pédagogiques, j'ai du mal à voir si on me parle d'une ville portuaire ou d'une agglomération particulière ; s'il est question d'une petite commune, je ne suis pas certain d'être dans une campagne vivante, dans un milieu périurbain en mutation ou en ruralité profonde. C'est ce que j'ai appelé des «projets hors sol ». Au départ, dans les études et diagnostics, on voit bien l'espace; puis, dans le document fondateur du projet éducatif, on voit de l'éducation dans le territoire, mais pas au territoire. Est-ce qu'un projet éducatif peut se pencher sur des enjeux territoriaux ou locaux? Doit-il rester purement éducatif ou peut-il aborder ces réalités et problématiques? On trouve parfois, dans l'annonce de certaines valeurs, les idées de citoyen critique et de changement social. Peut-on aller plus loin et concrétiser ces idées en les ancrant dans le territoire en question? Le projet pédagogique peut-il, lui aussi, s'ouvrir sur le territoire? On n'éduquerait plus à des thèmes, mais à des problèmes! Pour les associations dites d'environnement, cela semble logique, mais qu'en est-il pour une collectivité ? Un projet éducatif local contractualisé peut-il aborder les problèmes du milieu ? Certains diront qu'on est passé dans le politique et qu'on ne doit pas mélanger les choses!

Au côté des deux autres pôles de l'éducation à l'environnement que constituent le savoir et la personne, nous sommes ici dans le pôle écosocioculturel que nous appelons territoire. Cette notion d'éducation aux territoires nécessite partenariat, ouverture, transversalité et cohérence de nombreux lieux éducatifs (école, loisir, famille) et champs d'apprentissage (environnement, culture, science, tourisme, agriculture, 
consommation); or souvent, ces domaines sont séparés. Cela ne sera pas facile à réaliser, car les obstacles sont nombreux : découpage des services dans les structures, caractère contractuel des dispositifs, appel au bénévolat, etc. Le mot partenariat est souvent utilisé, mais que l'on soit optimiste, pessimiste ou réaliste, cette synergie est plus ou moins présente sur nos territoires. Et puis, quelle reconnaissance pour cette éducation hors l'école que certains appellent coéducation? On pourrait aussi parler d'éducation populaire! Quelle spécificité pour une ErE dans ces temps particuliers de vacances et de loisirs? L'ancrage local du CLSH en est sans doute une. Enfin, qui doit s'occuper de cette éducation aux territoires? Pour l'ErE, j'ai l'habitude de dire qu'il y a ceux qui font de l'ErE LEUR métier et ceux qui font de l'ErE DANS leur métier; alors, pour l'éducation relative aux territoires, je pense que c'est vraiment l'affaire de tous et en particulier, des agités du «glocal»!

\section{BIBLIOGRAPHIE}

Citéphile. (1998). Citadins et citoyens, un regard sur la ville. Actes du colloque tenu à Lille du 2 au 5 décembre 1998. La Madeleine : Citéphile.

Jacquemain, D. (1998). Dossier « Territoires ». Camaraderie, 244.

JPA. (2001). Actes des rencontres nationales «Éducation à l'environnement en centre de vacances et de loisirs ", 20-21 septembre 2001. Paris : JPA et Ministère de la Jeunesse, de l'Éducation nationale et de la Recherche.

Larue, D. (1998) De la nature aux territoires, le parcours des centres permanents d'initiatives pour l'environnement. Champs culturels, 8.

Lefèvre, H. (1974). La production de l'espace. Paris : Anthropos.

\section{NOTES}

1. En France, on appelle Centre de Vacances et de Loisirs (CVL) des accueils collectifs de mineurs (moins de 18 ans) pendant les vacances scolaires, les congés professionnels ou les loisirs. Ces accueils peuvent comporter un hébergement (centre de vacances) ou non (CLSH) et recouvrent des réalités variées selon les publics et les types d'organisation.

2. Un résumé de ces observations peut être consulté dans la revue Réussir (n50/51, 2003) : «Vers une éducation au développement durable ». Voir aussi les Actes du colloque environnements, cultures et développements de l'IFREE/ORE (Niort, 7-8 novembre 2002).

3. Cette vision recouvre aussi le domaine des activités scientifiques et la découverte des patrimoines.

4. Syndicat Intercommunal à Vocation Unique : ici, il s'agit de développer les loisirs des enfants et des jeunes.

5. « 1000 défis pour ma planète » est une opération nationale réunissant différents ministères (agriculture, environnement, éducation nationale, jeunesse et sports) qui labellisent et financent des projets associatifs. 
6. «Écologestes » est une opération menée sur les quatre départements bretons par l'association Bretagne Vivante (186 rue Anatole France, BP 32, 29276 Brest cedex) en partenariat avec la DRDJS, la Fédération bretonne des caisses d'allocation familiale et la Direction Régionale de l'Environnement.

\section{AUTEUR}

\section{HENRI LABBÉ}

Conseiller technique et pédagogique supérieur en matière d'activités scientifiques et d'éducation relative l'environnement à la Direction Régionale et Départementale de la Jeunesse et des Sports (DRDJS) de Bretagne depuis 1973, il accompagne, en parallèle, des mouvements et des réseaux nationaux et régionaux par des formations, des interventions, la rédaction d'articles et la conception d'outils pédagogiques. 